\title{
Climate Metrics for C1-C4 Hydrofluorocarbons (HFCs)
}

\author{
James B. Burkholder,* Paul Marshall, Partha P. Bera, Joseph S. Francisco, and Timothy J. Lee
}

Cite This: J. Phys. Chem. A 2020, 124, 4793-4800

Read Online

ABSTRACT: Hydrofluorocarbons (HFCs) are potent greenhouse gases that are potential substitutes for ozone depleting substances. The Kigali amendment lists 17 HFCs that are currently in commercial use to be regulated under the Montreal Protocol. Future commercial applications may explore the use of other HFCs, most of which currently lack an evaluation of their climate metrics. In this work, atmospheric lifetimes, radiative efficiencies (REs), global warming potentials (GWPs), and global temperature change potentials (GTPs) for all saturated HFCs with fewer than 5 carbon atoms are estimated to help guide future usage and policy decisions. Atmospheric lifetimes were estimated using a structure activity relationship (SAR) for $\mathrm{OH}$ radical reactivity and estimated $\mathrm{O}\left({ }^{1} \mathrm{D}\right)$ reactivity. Radiative metrics were obtained using theoret-

\section{HFC Climate Metrics A Theoretical Analysis of $<C 5$ Molecules}

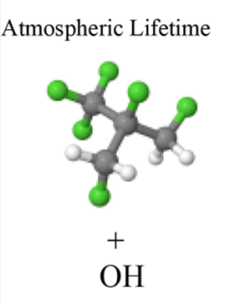

Global Warming Potential

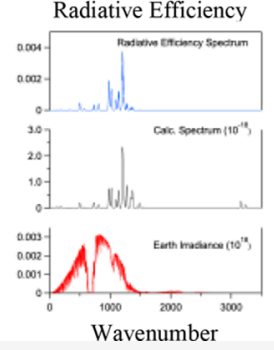

Global Warming Potential

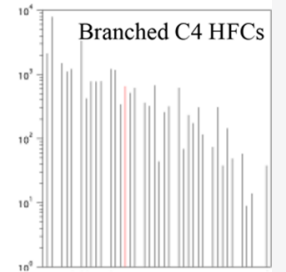
ically calculated infrared absorption spectra that were presented in a previous work. Calculations for some additional HFCs not included in the previous work were performed in this work. The HFCs display unique infrared spectra with strong absorption in the Earth's atmospheric infrared window region, primarily due to the $\mathrm{C}-\mathrm{F}$ stretching vibration. Results from this study show that the HFC global atmospheric lifetimes and REs are dependent upon their $\mathrm{H}$ atom content and molecular structure. Therefore, the HFC radiative metric evaluation requires a case-by-case evaluation. A thorough experimental evaluation of a targeted HFC's atmospheric lifetime and climate metrics is always highly recommended. However, in cases where it is experimentally difficult to separate isomers, the new results from this study should help guide the experiments, as well as provide relevant climate metrics with uncertainties and policy relevant data.

\section{INTRODUCTION}

Hydrofluorocarbons (HFCs) are, in general, potent greenhouse gases due to their strong infrared absorption in the Earth's atmospheric "window" region. HFCs are a current environmental concern because of their potential use as replacements for ozone depleting substances. Seventeen HFCs that are in commercial use today have been included in the Kigali amendment for regulation and phase-down under the Montreal Protocol (see Table 1). The atmospheric lifetimes and climate metrics (radiative efficiency (RE), global warming potential (GWP), and global temperature change potential (GTP)) for these HFCs have been defined based on detailed experimental studies of their $\mathrm{OH}$ reactivity and infrared absorption, as summarized in the World Meteorological Organization (WMO) ozone assessment report. ${ }^{1}$ In the future, commercial applications may wish to explore the use of other HFCs that are currently not included in the Montreal Protocol, nearly all of which lack a rigorous evaluation of their atmospheric lifetime and climate metrics.

In this work, estimates of atmospheric lifetimes, REs, GWPs, and GTPs for all HFCs with fewer than 5 carbon atoms are reported. Atmospheric lifetimes were estimated using a structure activity relationship (SAR) for $\mathrm{OH}$ radical reactivity and estimated $\mathrm{O}\left({ }^{1} \mathrm{D}\right)$ reactivity. Photolytic loss, which is expected to be primarily an upper-atmosphere loss process for HFCs, is negligible and was not evaluated as part of this work. Radiative metrics were determined using theoretically calculated infrared absorption spectra presented in the work of Bera et al. $^{2}$ and Kokkila et al. ${ }^{3}$ and supplemented in this work using the same theoretical methods. The calculated infrared spectra display strong absorption in the Earth's atmospheric infrared (IR) window region, primarily due to the $\mathrm{C}-\mathrm{F}$ stretching vibrational mode. The estimated lifetimes and radiative metrics for compounds with parameters listed in the WMO report, which are primarily based on experimental measurements, are used to validate our methods and provide a basis to estimate parameter uncertainties. The results from this study are intended to provide policy-relevant climate metrics for lightweight HFCs that are currently lacking quantitative experimental studies. This study does not, however, replace

Received: March 26, 2020

Revised: May 10, 2020

Published: May 11, 2020 
Table 1. Summary of C1-C2 Hydrofluorocarbon (HFC) Atmospheric Lifetimes and Climate Metrics ${ }^{a}$

\begin{tabular}{|c|c|c|c|c|c|c|c|c|c|c|c|}
\hline Formula & Abbrev. & CAS RN & $\begin{array}{c}\text { Total } \\
\text { Lifetime } \\
\text { (years) }\end{array}$ & $\begin{array}{c}\text { Tropospheric } \\
\text { OH Lifetime } \\
\text { (years) }\end{array}$ & $\begin{array}{l}\text { Stratospheric } \\
\text { Lifetime } \\
\text { (years) }\end{array}$ & $\mathrm{RE}$ & $\begin{array}{c}\text { GWP } \\
20\end{array}$ & $\begin{array}{c}\text { GWP } \\
100\end{array}$ & $\begin{array}{c}\text { GTP } \\
20\end{array}$ & $\begin{array}{c}\text { GTP } \\
50\end{array}$ & $\begin{array}{l}\text { GTP } \\
100\end{array}$ \\
\hline \multicolumn{12}{|l|}{ C1 Molecules } \\
\hline & & & & & & & & & & & \\
\hline $\mathrm{CHF}_{3}$ & HFC-23 & $75-46-7$ & 228 & 243 & 4420 & 0.18 & 11085 & 12690 & 11825 & 13340 & 13150 \\
\hline $\mathrm{CH}_{2} \mathrm{~F}_{2}$ & HFC-32 & $75-10-5$ & 5.4 & 5.5 & 124 & 0.11 & 2530 & 705 & 1440 & 154 & 98 \\
\hline $\mathrm{CH}_{3} \mathrm{~F}$ & HFC-41 & $593-53-3$ & 2.8 & 2.9 & 65 & 0.02 & 430 & 116 & 177 & 21 & 16 \\
\hline \multirow{2}{*}{\multicolumn{12}{|c|}{ C2 Molecules }} \\
\hline & & & & & & & & & & & \\
\hline $\mathrm{CHF}_{2}-\mathrm{CF}_{3}$ & HFC-125 & $354-33-6$ & 30 & 32 & 595 & 0.23 & 6280 & 3450 & 6040 & 3350 & 1180 \\
\hline $\mathrm{CHF}_{2}-\mathrm{CHF}_{2}$ & HFC-134 & $359-35-3$ & 10 & 10.5 & 240 & 0.19 & 3625 & 1135 & 2725 & 440 & 164 \\
\hline $\mathrm{CH}_{2} \mathrm{~F}-\mathrm{CF}_{3}$ & HFC-134a & $811-97-2$ & 14 & 14.1 & 267 & 0.16 & 3810 & 1360 & 3170 & 770 & 215 \\
\hline $\mathrm{CH}_{2} \mathrm{~F}-\mathrm{CHF}_{2}$ & HFC-143 & $430-66-0$ & 3.6 & 3.70 & 100 & 0.13 & 1250 & 340 & 580 & 64 & 48 \\
\hline $\mathrm{CH}_{3}-\mathrm{CF}_{3}$ & HFC-143a & $420-46-2$ & 51 & 57 & 612 & 0.16 & 7050 & 5080 & 7110 & 5390 & 2830 \\
\hline $\mathrm{CH}_{2} \mathrm{~F}-\mathrm{CH}_{2} \mathrm{~F}$ & HFC-152 & $624-72-6$ & 172 days & 172 days & 25 & 0.04 & 64 & 17 & 20 & 3.0 & 2.4 \\
\hline $\mathrm{CH}_{3}-\mathrm{CHF}_{2}$ & HFC-152a & $75-37-6$ & 1.6 & 1.55 & 39 & 0.10 & 545 & 148 & 190 & 26 & 21 \\
\hline $\mathrm{CH}_{3}-\mathrm{CH}_{2} \mathrm{~F}$ & HFC-161 & $353-36-6$ & 80 days & 80 days & 25 & 0.02 & 20 & 6 & 6 & $<1$ & $<1$ \\
\hline
\end{tabular}

${ }^{a_{T}}$ The gray shaded entries were taken from WMO (2018). The results from the present work for these HFCs are given in the Supporting Information datasheets. All other entries are from this work. The values reported in this work were obtained using methods consistent with the methods used to derive the WMO (2018) lifetimes and climate metrics: Radiative efficiency (RE) in units of $\mathrm{W} \mathrm{m}^{-2} \mathrm{ppb}^{-1}$; Global Warming Potential (GWP) and time-horizon; and Global Temperature Potential (GTP) and time-horizon.

the need for detailed experimental studies of specific targeted compounds prior to their commercial use.

\section{METHODOLOGY}

Atmospheric Lifetimes. The global HFC atmospheric lifetime $\left(\tau_{\text {atm }}\right)$ is defined as

$$
\frac{1}{\tau_{\mathrm{atm}}}=\frac{1}{\tau_{\mathrm{OH}}}+\frac{1}{\tau_{\mathrm{O}\left({ }^{1} \mathrm{D}\right)}}
$$

where $\tau_{\mathrm{OH}}$ and $\tau_{\mathrm{O}(1 \mathrm{D})}$ are the global lifetimes with respect to $\mathrm{OH}$ and $\mathrm{O}\left({ }^{1} \mathrm{D}\right)$ reactive loss, respectively. $\tau_{\text {atm }}$ can also be expressed in terms of its troposphere $\left(\tau_{\text {Trop }}\right)$, stratosphere $\left(\tau_{\text {Strat }}\right)$, and mesosphere $\left(\tau_{\text {Meso }}\right)$ lifetimes

$$
\frac{1}{\tau_{\text {atm }}}=\frac{1}{\tau_{\text {Trop }}}+\frac{1}{\tau_{\text {Strat }}}+\frac{1}{\tau_{\text {Meso }}}
$$

where, for example

$$
\frac{1}{\tau_{\text {Strat }}}=\frac{1}{\tau_{\text {Strat }}^{\mathrm{OH}}}+\frac{1}{\tau_{\text {Strat }}^{\mathrm{O}\left({ }^{1} \mathrm{D}\right)}}
$$

For the HFCs considered in this study, mesospheric loss is expected to be negligible and not considered further. In this work, $\tau_{\text {Trop }}^{\mathrm{OH}}$ was estimated using the $\mathrm{CH}_{3} \mathrm{CCl}_{3}(\mathrm{MCF})$ relative lifetime method ${ }^{4}$ where

$$
\tau_{\mathrm{Trop}}^{\mathrm{OH}}=\tau_{\mathrm{OH}}^{\mathrm{HFC}}=\frac{k_{\mathrm{MCF}}(272 \mathrm{~K})}{k_{\mathrm{HFC}}(272 \mathrm{~K})} \tau_{\mathrm{OH}}^{\mathrm{MCF}}
$$

with the MCF recommended $\mathrm{OH}$ reaction rate coefficient, $k_{\mathrm{MCF}}(272 \mathrm{~K})=6.14 \times 10^{-15} \mathrm{~cm}^{3}$ molecule $\mathrm{s}^{-1} \mathrm{~s}^{-15}$ and $\tau_{\mathrm{OH}}^{\mathrm{MCF}}$ is the recommended tropospheric lifetime of MCF, 6.1 years, ${ }^{4}$ due to reaction with the $\mathrm{OH}$ radical.

In the absence of experimentally determined $\mathrm{OH}$ reaction rate coefficients, the structure activity relationship (SAR) developed by DeMore ${ }^{6}$ was used to estimate $\mathrm{OH}$ reaction rate coefficients. For the determination of $k_{\mathrm{HFC}}(272 \mathrm{~K})$, the reaction rate coefficient $E / R$ was estimated based on the trend in Arrhenius parameters, $k(T)=A \exp (-E / R T)$, for the HFCs recommended in Burkholder et al.; ${ }^{5} E / R=\exp (1.9686$ $-0.15697 k(298 \mathrm{~K})) \mathrm{K}$. On the basis of a comparison with recommended rate coefficient data, the uncertainty in the SAR room temperature rate coefficients is estimated to be better than $30 \%$, as shown in Figure 1. The uncertainty in $k(272 \mathrm{~K})$

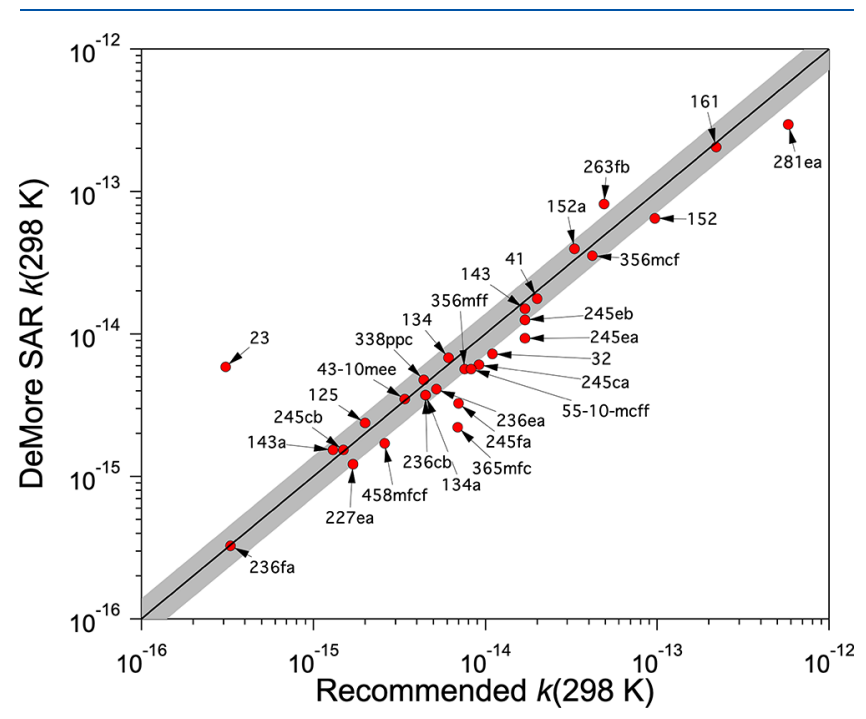

Figure 1. Correlation between the $298 \mathrm{~K} \mathrm{OH}+$ hydrofluorocarbon (HFC) reaction rate coefficients, $k(298 \mathrm{~K})$, calculated using the structure activity relationship (SAR) from DeMore ${ }^{6}$ and the recommended values from Burkholder et $\mathrm{al}^{5}$ (28 HFCs in total). The HFCs are labeled on the graph. The line is the 1:1 relationship. The edges of the gray shaded region represent the $\pm 30 \%$ range around the 1:1 line.

will, most likely, be greater due to the uncertainty in the assumed $E / R$ value. We estimate a $50 \%$ uncertainty in $k(272$ K) for all compounds in this study.

$\tau_{\text {Strat }}$ for the HFCs was determined from a combination of $\mathrm{OH}$ and $\mathrm{O}\left({ }^{1} \mathrm{D}\right)$ reactive loss. We estimated stratospheric $\mathrm{OH}$ loss lifetimes, $\tau_{\text {Strat }}^{\mathrm{OH}}$ following the methodology used in $\mathrm{WMO}-$ $2014^{1}$ and our previous study of HCFCs climate metrics. ${ }^{7}$ $\mathrm{O}\left({ }^{1} \mathrm{D}\right)$ rate coefficients were estimated using the reactivity trends reported in Baasandorj et al. ${ }^{8}$ In most cases, the stratospheric loss via the $\mathrm{OH}$ reaction accounts for approximately $\leq 5 \%$ of the total $\mathrm{OH}$ loss process. The $\mathrm{O}\left({ }^{1} \mathrm{D}\right)$ reaction represents only a minor contributor to the global loss of the HFCs, <2\%. A minimum stratospheric lifetime of 25 
Table 2. Summary of C3 Hydrofluorocarbon (HFC) Atmospheric Lifetimes and Climate Metrics ${ }^{a}$

\begin{tabular}{|c|c|c|c|c|c|c|c|c|c|c|c|}
\hline Formula & Abbrev. & CAS RN & $\begin{array}{c}\text { Total } \\
\text { Lifetime } \\
\text { (years) }\end{array}$ & $\begin{array}{c}\text { Tropospheric } \\
\text { OH Lifetime } \\
\text { (years) }\end{array}$ & $\begin{array}{c}\text { Stratospheric } \\
\text { Lifetime } \\
\text { (years) }\end{array}$ & RE & $\begin{array}{c}\text { GWP } \\
20\end{array}$ & $\begin{array}{c}\text { GWP } \\
100\end{array}$ & $\begin{array}{c}\text { GTP } \\
20\end{array}$ & $\begin{array}{c}\text { GTP } \\
50\end{array}$ & $\begin{array}{c}\text { GTP } \\
100\end{array}$ \\
\hline $\mathrm{CHF}_{2}-\mathrm{CF}_{2}-\mathrm{CF}_{3}$ & HFC-227ca & $2252-84-8$ & 30 & 32 & 640 & 0.27 & 5260 & 2865 & 5070 & 2795 & 975 \\
\hline $\mathrm{CF}_{3}-\mathrm{CHF}-\mathrm{CF}_{3}$ & HFC-227ea & $431-89-0$ & 36 & 37.5 & 673 & 0.26 & 5250 & 3140 & 5140 & 3180 & 1260 \\
\hline $\mathrm{CHF}_{2}-\mathrm{CF}_{2}-\mathrm{CHF}_{2}$ & HFC-236ca & $680-00-2$ & 11.4 & 11.9 & 268 & 0.304 & 4259 & 1399 & 3327 & 622 & 205 \\
\hline $\mathrm{CH}_{2} \mathrm{~F}-\mathrm{CF}_{2}-\mathrm{CF}_{3}$ & HFC-236cb & $677-56-5$ & 13.4 & 14 & 305 & 0.23 & 3540 & 1235 & 2915 & 670 & 192 \\
\hline $\mathrm{CHF}_{2}-\mathrm{CHF}-\mathrm{CF}_{3}$ & HFC-236ea & $431-63-0$ & 11.4 & 11.9 & 270 & 0.30 & 4190 & 1370 & 3290 & 620 & 202 \\
\hline $\mathrm{CF}_{3}-\mathrm{CH}_{2}-\mathrm{CF}_{3}$ & HFC-236fa & $690-39-1$ & 213 & 253 & 1350 & 0.24 & 6785 & 7680 & 7230 & 8090 & 7870 \\
\hline $\mathrm{CH}_{2} \mathrm{~F}-\mathrm{CF}_{2}-\mathrm{CHF}_{2}$ & HFC-245ca & $679-86-7$ & 6.6 & 6.9 & 165 & 0.24 & 2530 & 720 & 1600 & 180 & 102 \\
\hline $\mathrm{CH}_{3}-\mathrm{CF}_{2}-\mathrm{CF}_{3}$ & HFC-245cb & $1814-88-6$ & 39.9 & 43 & 550 & 0.24 & 6340 & 4000 & 6280 & 4150 & 1800 \\
\hline $\mathrm{CHF}_{2}$-CHF-CHF 2 & HFC-245ea & $24270-66-4$ & 3.2 & 3.3 & 95 & 0.16 & 860 & 233 & 375 & 44 & 32 \\
\hline $\mathrm{CH}_{2} \mathrm{~F}-\mathrm{CHF}-\mathrm{CF}_{3}$ & HFC-245eb & $431-31-2$ & 3.2 & 3.3 & 90 & 0.20 & 1070 & 290 & 460 & 54 & 40 \\
\hline $\mathrm{CHF}_{2}-\mathrm{CH}_{2}-\mathrm{CF}_{3}$ & HFC-245fa & $460-73-1$ & 7.9 & 8.2 & 149 & 0.24 & 2980 & 880 & 2040 & 260 & 124 \\
\hline $\mathrm{CH}_{2} \mathrm{~F}-\mathrm{CF}_{2}-\mathrm{CH}_{2} \mathrm{~F}$ & HFC-254ca & $813-75-2$ & 2.56 & 2.65 & 74.5 & 0.147 & 734 & 200 & 292 & 36 & 28 \\
\hline $\mathrm{CH}_{3}-\mathrm{CF}_{2}-\mathrm{CHF}_{2}$ & HFC-254cb & $40723-63-5$ & 10.8 & 11.4 & 228 & 0.203 & 3603 & 1164 & 2772 & 490 & 169 \\
\hline $\mathrm{CH}_{2} \mathrm{~F}-\mathrm{CHF}-\mathrm{CHF}_{2}$ & HFC-254ea & $24270-68-6$ & 1.94 & 2.01 & 59 & 0.173 & 655 & 178 & 239 & 31 & 25 \\
\hline $\mathrm{CH}_{3}-\mathrm{CHF}-\mathrm{CF}_{3}$ & HFC-254eb & $421-48-7$ & 2.25 & 2.33 & 67 & 0.178 & 782 & 213 & 298 & 38 & 29 \\
\hline $\mathrm{CHF}_{2}-\mathrm{CH}_{2}-\mathrm{CHF}_{2}$ & HFC-254fa & $66794-30-7$ & 3.99 & 4.14 & 107 & 0.236 & 1824 & 500 & 887 & 97 & 69 \\
\hline $\mathrm{CH}_{2} \mathrm{~F}-\mathrm{CH}_{2}-\mathrm{CF}_{3}$ & HFC-254fb & $460-36-6$ & 1.38 & 1.43 & 44 & 0.155 & 419 & 114 & 142 & 20 & 16 \\
\hline $\mathrm{CH}_{3}-\mathrm{CF}_{2}-\mathrm{CH}_{2} \mathrm{~F}$ & HFC-263ca & $811-94-9$ & 3.67 & 3.81 & 98 & 0.141 & 1187 & 324 & 554 & 62 & 45 \\
\hline $\mathrm{CH}_{2} \mathrm{~F}-\mathrm{CHF}-\mathrm{CH}_{2} \mathrm{~F}$ & HFC-263ea & $66794-36-3$ & 0.53 & 0.55 & 25 & 0.071 & 87 & 24 & 27 & 4 & 3 \\
\hline $\mathrm{CH}_{3}$-CHF-CHF 2 & HFC-263eb & $66794-35-2$ & 1.09 & 1.12 & 36 & 0.118 & 298 & 81 & 97 & 14 & 11 \\
\hline $\mathrm{CH}_{2} \mathrm{~F}-\mathrm{CH}_{2}-\mathrm{CHF}_{2}$ & HFC-263fa & $24270-67-5$ & 1.07 & 1.10 & 35 & 0.126 & 311 & 85 & 102 & 14 & 12 \\
\hline $\mathrm{CH}_{3}-\mathrm{CH}_{2}-\mathrm{CF}_{3}$ & HFC-263fb & $421-07-8$ & 1.1 & 1.16 & 40 & 0.10 & 250 & 68 & 83 & 12 & 9.5 \\
\hline $\mathrm{CH}_{3}-\mathrm{CF}_{2}-\mathrm{CH}_{3}$ & HFC-272ca & $420-45-1$ & 9 & 9.7 & 185 & 0.07 & 1580 & 480 & 1140 & 163 & 69 \\
\hline $\mathrm{CH}_{3}-\mathrm{CHF}-\mathrm{CH}_{2} \mathrm{~F}$ & HFC-272ea & $62126-90-3$ & 0.38 & 0.38 & 25 & 0.055 & 58 & 16 & 18 & 3 & 2 \\
\hline $\mathrm{CH}_{2} \mathrm{~F}-\mathrm{CH}_{2}-\mathrm{CH}_{2} \mathrm{~F}$ & HFC-272fa & $462-39-5$ & 0.19 & 0.19 & 25 & 0.037 & 20 & 5 & 6 & 1 & 1 \\
\hline $\mathrm{CH}_{3}-\mathrm{CH}_{2}-\mathrm{CHF}_{2}$ & HFC-272fb & $430-61-5$ & 0.71 & 0.73 & 25 & 0.077 & 153 & 42 & 48 & 7 & 6 \\
\hline $\mathrm{CH}_{3}-\mathrm{CHF}-\mathrm{CH}_{3}$ & HFC-281ea & $420-26-8$ & 27 days & 27 days & 25 & 0.019 & 11 & 3 & 3 & 0.50 & 0.40 \\
\hline $\mathrm{CH}_{3}-\mathrm{CH}_{2}-\mathrm{CH}_{2} \mathrm{~F}$ & HFC-281fa & $460-13-9$ & 0.13 & 0.13 & 25 & 0.016 & 8 & 2 & 2 & 0.35 & 0.29 \\
\hline
\end{tabular}

${ }^{a_{\text {The }}}$ gray shaded entries were taken from WMO (2018). The results from the present work for these HFCs are given in the Supporting Information datasheets. All other entries are from this work. The values reported in this work were obtained using methods consistent with the methods used to derive the WMO (2018) lifetimes and climate metrics: Radiative efficiency (RE) in units of $\mathrm{W} \mathrm{m}^{-2} \mathrm{ppb}^{-1}$; Global Warming Potential (GWP) and time-horizon; and Global Temperature Potential (GTP) and time-horizon.

years was applied to account for transport limited stratospheric lifetimes.

Quantum Chemistry Calculations. The theoretical methods used to calculate HFC infrared absorption spectra are taken from Bera et al. ${ }^{2}$ and Kokkila et al. ${ }^{3}$ Calculations were performed using second-order Møller-Plesset perturbation theory (MP2) using the frozen-core approximation together with a double- $\zeta$ plus polarization one-particle basis set that includes diffuse functions on all atoms, including $\mathrm{H}$, and is denoted DZP++. ${ }^{9-12}$ The present study includes branched C4 HFCs, which were not part of the Kokkila et al. ${ }^{3}$ study. The calculated spectra used in this work are for the lowest-energy structure obtained after very tight geometry optimizations and have been computed using the double harmonic approximation, following Kokkila et al.

The level of theory was evaluated based on comparison with available experimental HFC infrared spectra. Although the properties of each HFC may vary, as stated by Kokkila et al., on the basis of comparisons to experiment the theoretically computed infrared spectra band strengths are estimated to be between 5\% and 20\% larger than experiment. There has not been a systematic study comparing the $\mathrm{C}-\mathrm{F}$ stretching harmonic frequencies computed at the MP2/DZP++ level of theory with experimental fundamental vibrational frequencies, but there are at least two relevant studies that give us an idea of the uncertainty of the band centers. Simandiras et al. ${ }^{13}$ report MP2/DZP harmonic frequencies for fluoroethane and find that the $\mathrm{C}-\mathrm{F}$ stretching harmonic frequency is computed to be about $21 \mathrm{~cm}^{-1}$ higher than experiment, which amounts to an error of about $2.4 \%$. In this case, the majority of that error is probably the neglect of an anharmonic correction for the $\mathrm{C}-\mathrm{F}$ stretch. Second, Breidung et al., ${ }^{14}$ using the MP2/DZP level of theory, compute the symmetric and antisymmetric $\mathrm{C}-\mathrm{F}$ stretches of difluorethyne to be $22 \mathrm{~cm}^{-1}$ too low and $8 \mathrm{~cm}^{-1}$ too high compared to experimental fundamental vibrational frequencies, respectively. These amount to errors of $-2.8 \%$ and $0.6 \%$, respectively. Hence, for the majority of the HFCs included in this study, we would expect the band centers to be within $\pm 3 \%$ or better. All of the new calculations have been run with the Q-Chem 5 quantum chemistry package. ${ }^{15}$

Climate Metrics. Radiative efficiencies (REs) were calculated using the $298 \mathrm{~K}$ calculated infrared absorption spectra and the parameterization of the Earth's irradiance given in Hodnebrog et al. ${ }^{16}$ The calculated spectra were broadened using a Gaussian function with a fwhm (full width at halfmaximum) of $20 \mathrm{~cm}^{-1}$ to provide a more realistic representation of the spectrum band widths and overlap with Earth's irradiance profile. Note that the calculated infrared spectra include vibrational bands below $500 \mathrm{~cm}^{-1}$, which is usually the lower limit for experimental infrared absorption spectra measurements. The contribution of vibrational bands in this region is, however, usually minor, i.e., $<1 \%$.

Atmospheric lifetime-adjusted REs were calculated using the parameterization developed in Hodnebrog et al. ${ }^{16}$ for compounds dominated by tropospheric $\mathrm{OH}$ loss with a "CFC-11-like" emission distribution. A $+10 \%$ correction was 
Table 3. Summary of Linear-C4 Hydrofluorocarbon (HFC) Atmospheric Lifetimes and Climate Metrics ${ }^{a}$

\begin{tabular}{|c|c|c|c|c|c|c|c|c|c|c|c|}
\hline Formula & Abbrev. & CAS RN & $\begin{array}{c}\begin{array}{c}\text { Total } \\
\text { Lifetime } \\
\text { (years) }\end{array} \\
\end{array}$ & $\begin{array}{c}\text { Tropospheric } \\
\begin{array}{c}\text { OH Lifetime } \\
\text { (years) }\end{array}\end{array}$ & $\begin{array}{c}\text { Stratospheric } \\
\text { Lifetime } \\
\text { (years) }\end{array}$ & $\mathrm{RE}$ & $\begin{array}{c}\text { GWP } \\
20\end{array}$ & $\begin{array}{c}\text { GWP } \\
100\end{array}$ & $\begin{array}{c}\text { GTP } \\
20\end{array}$ & $\begin{array}{c}\text { GTP } \\
50\end{array}$ & $\begin{array}{l}\text { GTP } \\
100\end{array}$ \\
\hline $\mathrm{CHF}_{2}-\mathrm{CF}_{2}-\mathrm{CF}_{2}-\mathrm{CF}_{3}$ & HFC-329p & $375-17-7$ & 32 & 34 & 675 & 0.31 & 4720 & 2630 & 4565 & 2595 & 935 \\
\hline $\mathrm{CF}_{3}-\mathrm{CHF}-\mathrm{CF}_{2}-\mathrm{CF}_{3}$ & HFC-329me & $680-17-1$ & 48.2 & 50.9 & 885 & 0.328 & 5515 & 3862 & 5524 & 4067 & 2047 \\
\hline & & & & & & & & & & & \\
\hline $\mathrm{CH}_{2} \mathrm{~F}-\mathrm{CF}_{2}-\mathrm{CF}_{2}-\mathrm{CF}_{3}$ & HFC-338q & $662-35-1$ & 14.6 & 15.3 & 325 & 0.276 & 3369 & 1229 & 2831 & 728 & 197 \\
\hline $\mathrm{CHF}_{2}-\mathrm{CHF}_{-}-\mathrm{CF}_{2}-\mathrm{CF}_{3}$ & HFC-338mce & $119450-58-7$ & 9.27 & 9.66 & 228 & 0.305 & 2795 & 860 & 2034 & 300 & 122 \\
\hline $\mathrm{CHF}_{2}-\mathrm{CF}_{2}-\mathrm{CHF}-\mathrm{CF}_{3}$ & HFC-338mec & $35230-11-6$ & 11.7 & 12.2 & 274 & 0.322 & 3458 & 1149 & 2728 & 530 & 169 \\
\hline $\mathrm{CHF}_{2}-\mathrm{CF}_{2}-\mathrm{CF}_{2}-\mathrm{CHF}_{2}$ & HFC-338pcc & $377-36-6$ & 13.5 & 14.0 & 360 & 0.324 & 3407 & 1119 & 2662 & 498 & 164 \\
\hline $\mathrm{CF}_{3}-\mathrm{CH}_{2}-\mathrm{CF}_{2}-\mathrm{CF}_{3}$ & HFC-338mf & $2924-29-0$ & 184 & 214 & 1289 & 0.307 & 6514 & 7214 & 6894 & 7562 & 7115 \\
\hline $\mathrm{CF}_{3}-\mathrm{CHF}-\mathrm{CHF}-\mathrm{CF}_{3}$ & HFC-338mee & $75995-72-1$ & 11.4 & 11.9 & 269 & 0.338 & 3571 & 1175 & 2795 & 526 & 172 \\
\hline $\mathrm{CH}_{3}-\mathrm{CF}_{2}-\mathrm{CF}_{2}-\mathrm{CF}_{3}$ & HFC- $347 \mathrm{mcc}$ & $662-00-0$ & 36.8 & 39.5 & 529 & 0.253 & 4789 & 2902 & 4685 & 2938 & 1191 \\
\hline $\mathrm{CH}_{2} \mathrm{~F}-\mathrm{CHF}-\mathrm{CF}_{2}-\mathrm{CF}_{3}$ & HFC-347mce & $75995-85-6$ & 3.35 & 3.47 & 95 & 0.236 & 969 & 264 & 432 & 49 & 37 \\
\hline $\mathrm{CH}_{2} \mathrm{~F}_{-} \mathrm{CF}_{2}-\mathrm{CHF}-\mathrm{CF}_{3}$ & HFC-347mec & $53005-35-9$ & 4.22 & 4.37 & 116 & 0.264 & 1357 & 372 & 679 & 73 & 52 \\
\hline $\mathrm{CH}_{2} \mathrm{~F}-\mathrm{CF}_{2}-\mathrm{CF}_{2}-\mathrm{CHF}_{2}$ & HFC-347pcc & $119450-61-2$ & 8.70 & 9.08 & 205 & 0.287 & 2759 & 834 & 1958 & 271 & 118 \\
\hline $\mathrm{CHF}_{2}-\mathrm{CH}_{2}-\mathrm{CF}_{2}-\mathrm{CF}_{3}$ & HFC-347mcf & $161791-36-2$ & 8.64 & 9.02 & 204 & 0.326 & 3115 & 940 & 2205 & 303 & 133 \\
\hline $\mathrm{CHF}_{2}-\mathrm{CHF}-\mathrm{CHF}-\mathrm{CF}_{3}$ & HFC-347mee & $151868-61-0$ & 5.00 & 5.20 & 133 & 0.298 & 1795 & 497 & 981 & 104 & 69 \\
\hline $\mathrm{CHF}_{2}-\mathrm{CHF}_{-} \mathrm{CF}_{2}-\mathrm{CHF}_{2}$ & HFC-347pce & $119450-64-5$ & 6.43 & 6.70 & 162 & 0.288 & 2176 & 620 & 1351 & 151 & 87 \\
\hline $\mathrm{CHF}_{2}-\mathrm{CF}_{2}-\mathrm{CH}_{2}-\mathrm{CF}_{3}$ & HFC- $347 \mathrm{mfc}$ & $119450-65-6$ & 14.6 & 15.4 & 300 & 0.308 & 4118 & 1502 & 3459 & 888 & 241 \\
\hline $\mathrm{CF}_{3}-\mathrm{CH}_{2}-\mathrm{CHF}-\mathrm{CF}_{3}$ & HFC-347mef & $86884-16-4$ & 8.53 & 8.90 & 202 & 0.318 & 3010 & 906 & 2119 & 288 & 128 \\
\hline $\mathrm{CH}_{3}-\mathrm{CHF}-\mathrm{CF}_{2}-\mathrm{CF}_{3}$ & HFC-356mce & $161791-32-8$ & 2.25 & 2.33 & 67 & 0.204 & 626 & 170 & 239 & 30 & 24 \\
\hline $\mathrm{CH}_{3}-\mathrm{CF}_{2}-\mathrm{CHF}-\mathrm{CF}_{3}$ & $\mathrm{HFC}-356 \mathrm{mec}$ & $76523-97-2$ & 13.9 & 14.6 & 268 & 0.251 & 3623 & 1290 & 3001 & 720 & 201 \\
\hline $\mathrm{CH}_{3}-\mathrm{CF}_{2}-\mathrm{CF}_{2}-\mathrm{CHF}_{2}$ & HFC-356pcc & $119450-66-7$ & 10.8 & 11.4 & 228 & 0.262 & 3254 & 1051 & 2503 & 442 & 152 \\
\hline $\mathrm{CH}_{2} \mathrm{~F}-\mathrm{CH}_{2}-\mathrm{CF}_{2}-\mathrm{CF}_{3}$ & HFC- $356 \mathrm{mcf}$ & $161791-33-9$ & 1.2 & 1.26 & 40 & 0.214 & 404 & 110 & 137 & 19 & 15 \\
\hline $\mathrm{CH}_{2} \mathrm{~F}-\mathrm{CHF}-\mathrm{CHF}-\mathrm{CF}_{3}$ & HFC-356mee & $119450-67-8$ & 2.21 & 2.28 & 66 & 0.220 & 661 & 180 & 251 & 32 & 25 \\
\hline $\mathrm{CH}_{2} \mathrm{~F}-\mathrm{CHF}-\mathrm{CF}_{2}-\mathrm{CHF}_{2}$ & HFC-356pce & $119450-68-9$ & 2.87 & 2.98 & 82 & 0.220 & 859 & 234 & 358 & 43 & 32 \\
\hline $\mathrm{CH}_{2} \mathrm{~F}_{-} \mathrm{CF}_{2}-\mathrm{CH}_{2}-\mathrm{CF}_{3}$ & HFC-356mfc & $76546-55-9$ & 4.84 & 5.03 & 125 & 0.249 & 1611 & 445 & 866 & 92 & 62 \\
\hline $\mathrm{CH}_{2} \mathrm{~F}-\mathrm{CF}_{2}-\mathrm{CHF}-\mathrm{CHF}_{2}$ & HFC-356pec & $114810-03-6$ & 2.83 & 2.93 & 81 & 0.228 & 878 & 239 & 364 & 44 & 33 \\
\hline $\mathrm{CHF}_{2}-\mathrm{CH}_{2}-\mathrm{CHF}_{-} \mathrm{CF}_{3}$ & HFC-356mef & $158421-88-6$ & 2.71 & 2.81 & 78 & 0.260 & 961 & 262 & 391 & 47 & 36 \\
\hline $\mathrm{CHF}_{2}-\mathrm{CHF}-\mathrm{CH}_{2}-\mathrm{CF}_{3}$ & HFC-356mfe & $76523-98-3$ & 3.05 & 3.17 & 86 & 0.263 & 1093 & 298 & 468 & 55 & 41 \\
\hline $\mathrm{CHF}_{2}-\mathrm{CH}_{2}-\mathrm{CF}_{2}-\mathrm{CHF}_{2}$ & HFC-356pcf & $119450-69-0$ & 5.43 & 5.66 & 137 & 0.270 & 1946 & 543 & 1111 & 119 & 76 \\
\hline $\mathrm{CHF}_{2}$-CHF-CHF-CHF 2 & HFC-356pee & $392-45-0$ & 3.17 & 3.29 & 89 & 0.251 & 1081 & 295 & 470 & 55 & 41 \\
\hline $\mathrm{CF}_{3}-\mathrm{CH}_{2}-\mathrm{CH}_{2}-\mathrm{CF}_{3}$ & HFC-356mff & $407-59-0$ & 8.5 & 8.9 & 190 & 0.300 & 3383 & 1045 & 2474 & 371 & 149 \\
\hline $\mathrm{CH}_{2} \mathrm{~F}-\mathrm{CF}_{2}-\mathrm{CF}_{2}-\mathrm{CH}_{2} \mathrm{~F}$ & HFC-356qcc & $114810-02-5$ & 7.03 & 7.34 & 166 & 0.239 & 2154 & 622 & 1395 & 163 & 87 \\
\hline $\mathrm{CH}_{3}-\mathrm{CH}_{2}-\mathrm{CF}_{2}-\mathrm{CF}_{3}$ & HFC-365mcf & $37826-35-0$ & 1.64 & 1.69 & 50 & 0.193 & 483 & 131 & 169 & 23 & 18 \\
\hline $\mathrm{CH}_{3}-\mathrm{CHF}-\mathrm{CHF}-\mathrm{CF}_{3}$ & HFC-365mee & $161791-22-6$ & 1.09 & 1.12 & 36 & 0.174 & 289 & 79 & 94 & 13 & 11 \\
\hline $\mathrm{CH}_{3}-\mathrm{CHF}_{-}-\mathrm{CF}_{2}-\mathrm{CHF}_{2}$ & HFC-365pce & $158421-89-7$ & 4.35 & 4.52 & 112 & 0.203 & 1333 & 366 & 677 & 73 & 51 \\
\hline $\mathrm{CH}_{3}-\mathrm{CF}_{2}-\mathrm{CHF}-\mathrm{CHF}_{2}$ & HFC-365pec & $119450-71-4$ & 5.44 & 5.67 & 132 & 0.211 & 1710 & 477 & 976 & 104 & 67 \\
\hline $\mathrm{CH}_{3}-\mathrm{CF}_{2}-\mathrm{CF}_{2}-\mathrm{CH}_{2} \mathrm{~F}$ & HFC-365qcc & $119450-72-5$ & 10.1 & 10.6 & 205 & 0.222 & 2952 & 932 & 2216 & 360 & 134 \\
\hline $\mathrm{CH}_{3}-\mathrm{CF}_{2}-\mathrm{CH}_{2}-\mathrm{CF}_{3}$ & HFC- $365 \mathrm{mfc}$ & $406-58-6$ & 10.9 & 11.4 & 215 & 0.215 & 3005 & 972 & 2315 & 410 & 141 \\
\hline $\mathrm{CH}_{2} \mathrm{~F}-\mathrm{CH}_{2}-\mathrm{CHF}-\mathrm{CF}_{3}$ & HFC-365mef & 161791-23-7 & 1.07 & 1.10 & 35 & 0.180 & 294 & 80 & 96 & 14 & 11 \\
\hline $\mathrm{CH}_{2} \mathrm{~F}-\mathrm{CH}_{2}-\mathrm{CF}_{2}-\mathrm{CHF}_{2}$ & HFC-365pcf & $161791-25-9$ & 1.26 & 1.30 & 40 & 0.180 & 347 & 94 & 116 & 16 & 13 \\
\hline $\mathrm{CH}_{2} \mathrm{~F}-\mathrm{CHF}-\mathrm{CH}_{2}-\mathrm{CF}_{3}$ & HFC- $365 \mathrm{mfe}$ & 161791-24-8 & 0.84 & 0.87 & 28 & 0.167 & 214 & 58 & 68 & 10 & 8 \\
\hline $\mathrm{CH}_{2} \mathrm{~F}-\mathrm{CHF}-\mathrm{CHF}-\mathrm{CHF}_{2}$ & HFC-365qee & $157016-17-6$ & 1.96 & 2.03 & 59 & 0.178 & 535 & 146 & 196 & 26 & 20 \\
\hline $\mathrm{CH}_{2} \mathrm{~F}-\mathrm{CF}_{2}-\mathrm{CH}_{2}-\mathrm{CHF}_{2}$ & HFC-365pfc & $119450-76-9$ & 3.28 & 3.40 & 89 & 0.208 & 1041 & 284 & 460 & 53 & 39 \\
\hline $\mathrm{CH}_{2} \mathrm{~F}-\mathrm{CF}_{2}-\mathrm{CHF}-\mathrm{CH}_{2} \mathrm{~F}$ & HFC-365qce & $119450-75-8$ & 2.07 & 2.15 & 62 & 0.166 & 526 & 143 & 196 & 25 & 20 \\
\hline $\mathrm{CHF}_{2}-\mathrm{CH}_{2}-\mathrm{CH}_{2}-\mathrm{CF}_{3}$ & HFC- $365 \mathrm{mff}$ & $161879-85-2$ & 3.32 & 3.44 & 90 & 0.250 & 1263 & 344 & 561 & 64 & 48 \\
\hline $\mathrm{CHF}_{2}-\mathrm{CH}_{2}-\mathrm{CHF}-\mathrm{CHF}_{2}$ & HFC-365pef & $119450-77-0$ & 2.14 & 2.22 & 63 & 0.214 & 699 & 190 & 262 & 34 & 26 \\
\hline $\mathrm{CF}_{3}-\mathrm{CHF}-\mathrm{CH}_{2}-\mathrm{CH}_{3}$ & HFC-374mef & 161791-15-7 & 0.63 & 0.65 & 25 & 0.135 & 148 & 40 & 46 & 7 & 6 \\
\hline $\mathrm{CF}_{3}-\mathrm{CH}_{2}-\mathrm{CHF}-\mathrm{CH}_{3}$ & HFC-374mfe & $86884-13-1$ & 0.49 & 0.50 & 25 & 0.124 & 106 & 29 & 32 & 5 & 4 \\
\hline $\mathrm{CF}_{3}-\mathrm{CH}_{2}-\mathrm{CH}_{2}-\mathrm{CH}_{2} \mathrm{~F}$ & HFC-374mff & $83234-21-3$ & 0.21 & 0.21 & 25 & 0.078 & 29 & 8 & 9 & 1 & 1 \\
\hline $\mathrm{CHF}_{2}-\mathrm{CF}_{2}-\mathrm{CH}_{2}-\mathrm{CH}_{3}$ & HFC-374pcf & 143969-51-1 & 0.79 & 0.81 & 27 & 0.136 & 187 & 51 & 59 & 9 & 7 \\
\hline $\mathrm{CHF}_{2}$-CHF-CHF-CH & HFC-374pee & 161791-16-8 & 0.91 & 0.94 & 30 & 0.125 & 198 & 54 & 63 & 9 & 7 \\
\hline $\mathrm{CHF}_{2}-\mathrm{CHF}-\mathrm{CH}_{2}-\mathrm{CH}_{2} \mathrm{~F}$ & HFC-374pef & $161791-17-9$ & 0.86 & 0.89 & 29 & 0.137 & 205 & 56 & 65 & 9 & 8 \\
\hline $\mathrm{CHF}_{2}-\mathrm{CH}_{2}-\mathrm{CF}_{2}-\mathrm{CH}_{3}$ & HFC-374pfc & $625-09-2$ & 3.00 & 3.12 & 82 & 0.178 & 926 & 252 & 394 & 46 & 35 \\
\hline $\mathrm{CHF}_{2}-\mathrm{CH}_{2}-\mathrm{CHF}-\mathrm{CH}_{2} \mathrm{~F}$ & HFC-374pfe & 161791-18-0 & 0.75 & 0.77 & 26 & 0.126 & 164 & 45 & 51 & 8 & 6 \\
\hline $\mathrm{CH}_{2} \mathrm{~F}-\mathrm{CF}_{2}-\mathrm{CHF}-\mathrm{CH}_{3}$ & HFC-374qce & 161791-20-4 & 1.54 & 1.59 & 47 & 0.141 & 377 & 103 & 130 & 18 & 14 \\
\hline $\mathrm{CH}_{2} \mathrm{~F}-\mathrm{CHF}-\mathrm{CF}_{2}-\mathrm{CH}_{3}$ & HFC-374qec & $161791-19-1$ & 2.29 & 2.37 & 66 & 0.151 & 601 & 163 & 230 & 29 & 23 \\
\hline $\mathrm{CH}_{2} \mathrm{~F}-\mathrm{CF}_{2}-\mathrm{CH}_{2}-\mathrm{CH}_{2} \mathrm{~F}$ & HFC-374qcf & $161791-21-5$ & 1.07 & 1.11 & 35 & 0.133 & 248 & 68 & 81 & 12 & 9 \\
\hline $\mathrm{CH}_{2} \mathrm{~F}-\mathrm{CHF}-\mathrm{CHF}-\mathrm{CH}_{2} \mathrm{~F}$ & HFC-374qee & $119382-47-7$ & 1.20 & 1.24 & 38 & 0.121 & 252 & 69 & 84 & 12 & 9 \\
\hline $\mathrm{CH}_{3}-\mathrm{CF}_{2}-\mathrm{CF}_{2}-\mathrm{CH}_{3}$ & HFC-374scc & $421-74-9$ & 17.6 & 18.8 & 268 & 0.212 & 4410 & 1759 & 3865 & 1237 & 321 \\
\hline $\mathrm{CHF}_{2}-\mathrm{CH}_{2}-\mathrm{CH}_{2}-\mathrm{CHF}_{2}$ & HFC-374pff & $161879-84-1$ & 1.38 & 1.43 & 43 & 0.179 & 432 & 117 & 146 & 20 & 16 \\
\hline $\mathrm{CH}_{3}-\mathrm{CH}_{2}-\mathrm{CH}_{2}-\mathrm{CF}_{3}$ & HFC-383m & $460-34-4$ & 0.19 & 0.19 & 25 & 0.064 & 25 & 7 & 7 & 1 & 1 \\
\hline $\mathrm{CHF}_{2}-\mathrm{CHF}-\mathrm{CH}_{2}-\mathrm{CH}_{3}$ & HFC-383pe & $66675-41-0$ & 0.48 & 0.49 & 25 & 0.090 & 88 & 24 & 27 & 4 & 3 \\
\hline $\mathrm{CHF}_{2}-\mathrm{CH}_{2}-\mathrm{CHF}-\mathrm{CH}_{3}$ & HFC-383pfe & $66675-42-1$ & 0.45 & 0.46 & 25 & 0.095 & 86 & 23 & 26 & 4 & 3 \\
\hline $\mathrm{CHF}_{2}-\mathrm{CH}_{2}-\mathrm{CH}_{2}-\mathrm{CH}_{2} \mathrm{~F}$ & HFC-383pff & $66587-70-0$ & 0.20 & 0.20 & 25 & 0.099 & 40 & 11 & 12 & 2 & 1 \\
\hline $\mathrm{CH}_{2} \mathrm{~F}-\mathrm{CF}_{2}-\mathrm{CH}_{2}-\mathrm{CH}_{3}$ & HFC-383qcf & $66587-71-1$ & 1.22 & 1.26 & 38 & 0.110 & 270 & 73 & 89 & 13 & 10 \\
\hline $\mathrm{CH}_{2} \mathrm{~F}-\mathrm{CHF}-\mathrm{CHF}-\mathrm{CH}_{3}$ & HFC-383qee & $66587-72-2$ & 0.37 & 0.37 & 25 & 0.066 & 48 & 13 & 15 & 2 & 2 \\
\hline $\mathrm{CH}_{2} \mathrm{~F}-\mathrm{CHF}-\mathrm{CH}_{2}-\mathrm{CH}_{2} \mathrm{~F}$ & HFC-383qef & $66587-73-3$ & 0.49 & 0.50 & 25 & 0.071 & 71 & 19 & 22 & 3 & 3 \\
\hline $\mathrm{CH}_{2} \mathrm{~F}-\mathrm{CH}_{2}-\mathrm{CF}_{2}-\mathrm{CH}_{3}$ & HFC-383qfe & $66587-74-4$ & 1.10 & 1.14 & 35 & 0.108 & 241 & 66 & 79 & 11 & 9 \\
\hline $\mathrm{CH}_{3}-\mathrm{CF}_{2}-\mathrm{CHF}-\mathrm{CH}_{3}$ & HFC-383sce & $66587-75-5$ & 1.19 & 1.23 & 38 & 0.118 & 283 & 77 & 94 & 13 & 11 \\
\hline $\mathrm{CH}_{3}-\mathrm{CH}_{2}-\mathrm{CH}_{2}-\mathrm{CHF}_{2}$ & HFC-392pff & $2358-38-5$ & 0.17 & 0.17 & 25 & 0.037 & 15 & 4 & 4 & 1 & 1 \\
\hline $\mathrm{CH}_{3}-\mathrm{CH}_{2}-\mathrm{CHF}-\mathrm{CH}_{2} \mathrm{~F}$ & HFC-392gef & $686-65-7$ & 0.28 & 0.28 & 25 & 0.040 & 26 & 7 & 8 & 1 & 1 \\
\hline $\mathrm{CH}_{3}-\mathrm{CHF}-\mathrm{CH}_{2}-\mathrm{CH}_{2} \mathrm{~F}$ & HFC-392qfe & $691-42-9$ & 0.31 & 0.32 & 25 & 0.044 & 33 & 9 & 10 & 1 & 1 \\
\hline $\mathrm{CH}_{2} \mathrm{~F}-\mathrm{CH}_{2}-\mathrm{CH}_{2}-\mathrm{CH}_{2} \mathrm{~F}$ & HFC-392qff & $372-90-7$ & 0.11 & 0.11 & 25 & 0.026 & 7 & 2 & 2 & 0 & 0 \\
\hline $\mathrm{CH}_{3}-\mathrm{CH}_{2}-\mathrm{CF}_{2}-\mathrm{CH}_{3}$ & HFC-392scf & $353-81-1$ & 0.78 & 0.80 & 26 & 0.080 & 150 & 41 & 47 & 7 & 6 \\
\hline $\mathrm{CH}_{3}-\mathrm{CHF}-\mathrm{CHF}-\mathrm{CH}_{3}$ & HFC-392see & $666-21-7$ & 0.28 & 0.28 & 25 & 0.045 & 30 & 8 & 9 & 1 & 1 \\
\hline $\mathrm{CH}_{3}-\mathrm{CH}_{2}-\mathrm{CH}_{2}-\mathrm{CH}_{2} \mathrm{~F}$ & HFC-3-10-1q & $2366-52-1$ & 0.08 & 0.08 & 25 & 0.011 & 3 & 1 & 1 & 0 & 0 \\
\hline $\mathrm{CH}_{3}-\mathrm{CH}_{2}-\mathrm{CHF}-\mathrm{CH}_{3}$ & HFC-3-10-1se & $359-01-3$ & 0.10 & 0.10 & 25 & 0.014 & 4 & 1 & 1 & 0 & 0 \\
\hline
\end{tabular}

${ }^{a_{\text {The }}}$ gray shaded entries were taken from WMO (2018). The results from the present work for these HFCs are given in the Supporting Information datasheets. All other entries are from this work. The values reported in this work were obtained using methods consistent with the methods used to derive the WMO (2018) lifetimes and climate metrics: Radiative efficiency (RE) in units of $\mathrm{W} \mathrm{m}^{-2} \mathrm{ppb}^{-1}$; Global Warming Potential (GWP) and time-horizon; and Global Temperature Potential (GTP) and time-horizon. 
Table 4. Summary of Branched-C4 Hydrofluorocarbon (HFC) Atmospheric Lifetimes and Climate Metrics ${ }^{a}$

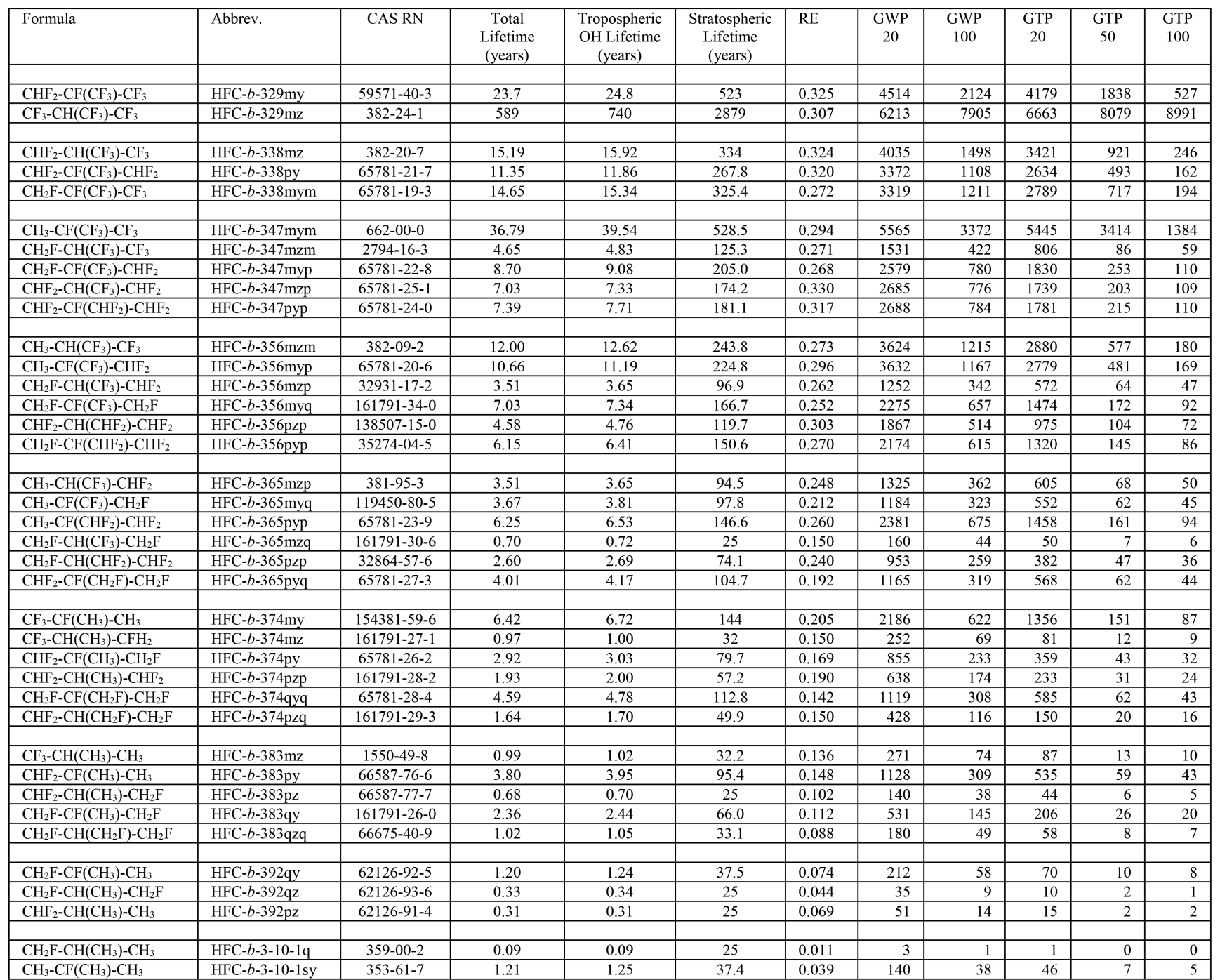

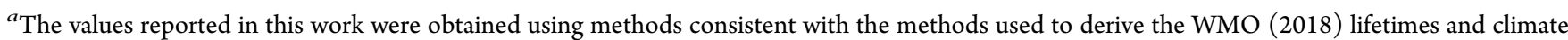
metrics: Radiative efficiency (RE) in units of $\mathrm{W} \mathrm{m}^{-2} \mathrm{ppb}^{-1}$; Global Warming Potential (GWP) and time-horizon; and Global Temperature Potential (GTP) and time-horizon.

applied to all molecules to account for a stratospheric temperature correction.

Global warming potentials on the 20- and 100-year time horizons $(T)$ were calculated relative to $\mathrm{CO}_{2}$ using

$$
\operatorname{GWP}(T)=\frac{\operatorname{RE} \tau\left[1-\exp \left(-T / \tau_{\mathrm{Atm}}\right)\right]}{\operatorname{Int} \mathrm{RF}_{\mathrm{CO}_{2}}(T)}
$$

The integrated (Int) $\mathrm{CO}_{2}$ radiative forcing term in the denominator is consistent with the GWP values reported in the WMO-2018 ${ }^{1}$ and IPCC-2013 ${ }^{17}$ assessments corresponding to a $\mathrm{CO}_{2}$ abundance of $391 \mathrm{ppm}$. Therefore, values reported in this work can be compared directly to values reported in the WMO and IPCC assessments.

Global temperature change potentials were calculated for the 20-, 50-, and 100-year time horizons using the parameterizations given in the IPCC $^{17}$ Supporting Information section S8.13.

\section{RESULTS AND DISCUSSION}

Lifetime and Climate Metric Data. Tables $1-4$ and the Supporting Information datasheets provide a summary of the lifetime, RE, GWP, and GTP results obtained in this study for $\mathrm{C} 1$ and $\mathrm{C} 2$, C3, linear-C4, and branched-C4 HFCs, respectively. Detailed individual datasheets for each HFC are given in the Supporting Information. The datasheets include $\mathrm{OH}$ and $\mathrm{O}\left({ }^{1} \mathrm{D}\right)$ rate coefficient results, a breakdown of partial and global lifetimes, and a comparison with literature and recommended values for all parameters where available. The results of this study are also presented in graphical form in Figures $\mathrm{S} 1-\mathrm{S} 4$ for the $\mathrm{C} 1$ and $\mathrm{C} 2, \mathrm{C} 3$, linear-C4, and branched-C4 HFCs, respectively. The shaded values in Tables 1-3 were taken from WMO-2018, ${ }^{1}$ while the values derived using our computational methods are provided in the datasheets. As expected, many of the HFCs are potent greenhouse gases (note that all RE values discussed in this section are lifetime and stratospheric temperature adjusted values). In addition to HFC isomers having different reactivity (lifetimes), each isomer has a unique infrared absorption 
spectrum and, thus, a unique RE. The HFCs with the highest $\mathrm{H}$ atom content generally have lower REs due to weaker infrared absorption in the Earth's atmospheric window region as well as shorter atmospheric lifetimes (REs reported in Tables 1-4 are lifetime corrected values).

We need to keep in perspective the level of uncertainty in the lifetimes and climate metrics given in the recommended values taken from the WMO assessment and the values from the present work given in Tables 1-4. The absolute uncertainty in the climate metrics may be substantial. The overall uncertainty includes contributions from laboratory kinetic measurements, which can be measured to $\sim 5-10 \%$ accuracy, and infrared absorption spectra, which can be measured to within 2-5\% accuracy. The RE determination parameterizations ${ }^{16}$ (irradiance, lifetime correction, and stratospheric temperature correction) contribute an estimated additional $25 \%$ uncertainty, or greater. Therefore, for the best of circumstances, GWPs are expected to have absolute uncertainties of $\sim 30 \%$. A comparison of relative metrics is expected to be more certain due to cancellation of errors. For example, the uncertainty in the $\mathrm{CO}_{2}$ forcing and irradiance parameterization would mostly cancel in a relative comparison. The relative uncertainties are most likely in the $10-15 \%$ range, or less.

We have not attempted to evaluate the uncertainty for each individual HFC included in this study. The agreement between the recommended RE and GWP climate metrics for the compounds included in WMO-2018 and those calculated here is shown in Figures 2 and 3, respectively. Note that the

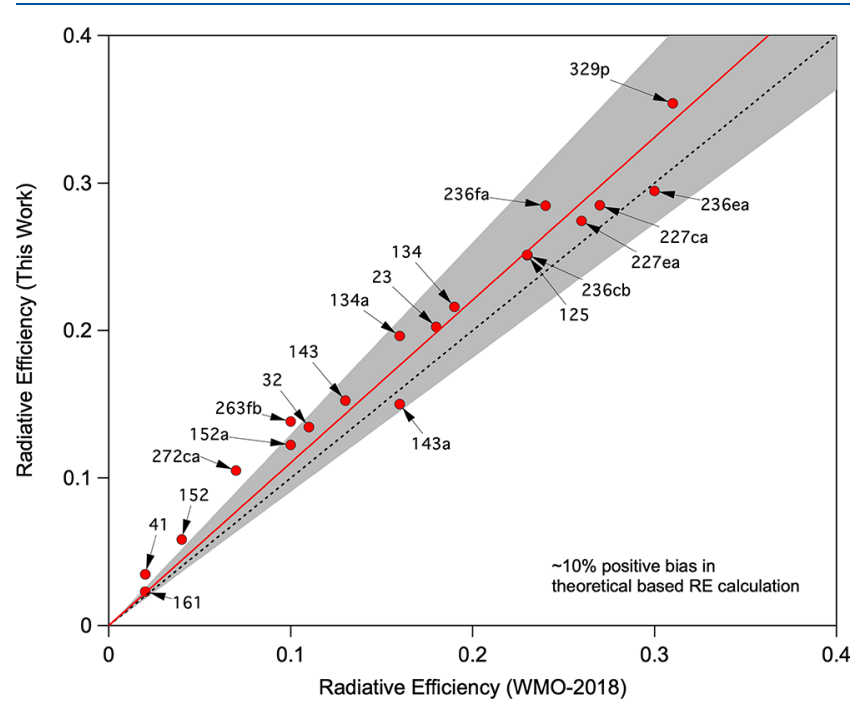

Figure 2. Comparison of hydrofluorcarbon (HFC) radiative efficiencies (REs) calculated in this work with those reported in WMO-2018. ${ }^{1}$ The HFCs used in the comparison are labeled on the graph. The dashed line is the 1:1 relationship. The solid red line is an unweighted linear least-squares fit to the data that shows a $10 \%$ positive bias in the values obtained in this study. The edges of the gray shaded region represent the $\pm 30 \%$ range around the fit line.

calculated metrics for HFC-23 are significant outliers due to the fact that the SAR does not reproduce the experimental $\mathrm{OH}$ reaction rate coefficient very well, as noted by DeMore. ${ }^{6}$ Overall, the comparison of RE values is very good with only slight positive biases. The predominant atmospheric loss process for HFCs is reaction with the $\mathrm{OH}$ radical. The DeMore $^{6}$ SAR reproduces the experimentally measured room

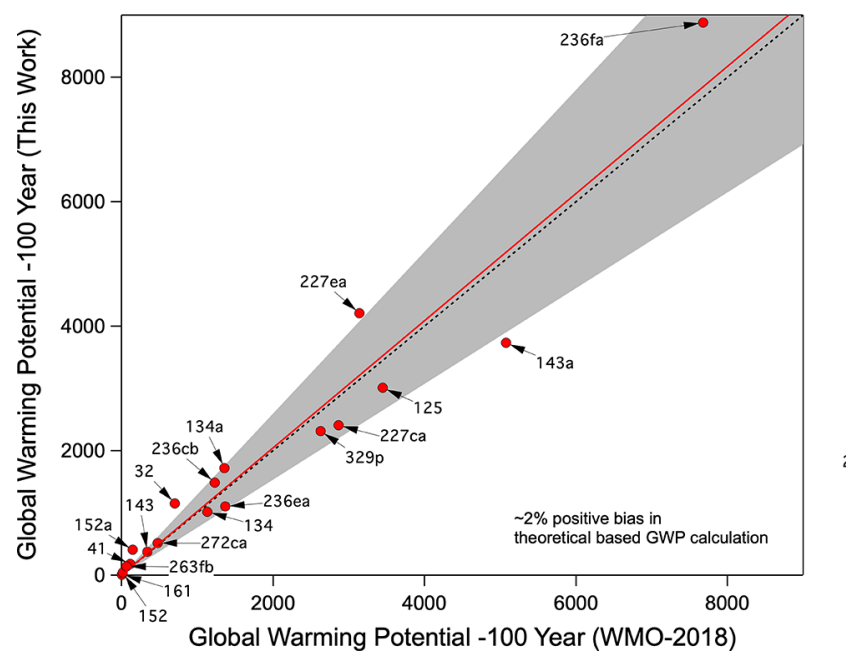

Figure 3. Comparison of hydrofluorcarbon (HFC) global warming potentials (GWPs) on the 100-year time horizon calculated in this work with those reported in WMO-2018. ${ }^{1}$ The HFCs used in the comparison are labeled on the graph. Note that the data point for HFC-23 falls well off the line and is not shown (see the SI datasheet for HFC-23 for values). The dashed line is the 1:1 relationship. The solid red line is an unweighted linear least-squares fit to the data shown that shows a $2 \%$ positive bias in the values obtained in this study. The edges of the gray shaded region represent the $\pm 30 \%$ range around the fit line.

temperature $\mathrm{OH}$ rate coefficients to within $\sim 30 \%$. We have conservatively estimated the uncertainty in $k(272 \mathrm{~K})$ to be $\sim 50 \%$. The uncertainty in the $\mathrm{OH}$ rate coefficient translates directly to the uncertainty in the HFC tropospheric lifetime. The theoretically derived REs agree to within $\sim 10 \%$ on average with the values derived using the experimentally measured spectra. As stated earlier, we estimated the uncertainty in the computed band strengths to be between $5 \%$ and $20 \%$ high, which is consistent with the theoretically determined REs in Figure 2 having $\sim 10 \%$ positive bias. For compounds with a lifetime of 0.2 to 1 year, i.e., those that fall on the steep portion of the lifetime correction profile given in Hodnebrog et al., ${ }^{16}$ additional significant uncertainty is introduced.

Figure 3 shows a comparison of the theoretically derived GWPs $(100)$ and those reported in WMO-2018. As expected, since the theoretically based REs exhibit $\sim 10 \%$ positive bias, the computationally based GWPs(100) show a slight positive bias, in this case only $\sim 2 \%$, which is better than should be expected. We conservatively estimate the climate metrics given in Tables $1-4$ to have uncertainties within a factor of 1.5 to 2, primarily depending on the lifetime of the HFC, i.e., compounds with a shorter lifetime are expected to have metrics with a greater degree of uncertainty.

Trends in HFC Metrics. Tables $1-4$ show that, in general, the substitution of $\mathrm{F}$ for $\mathrm{H}$ results in reduced $\mathrm{OH}$ radical reactivity, which is consistent with the enhancement factors in the $\mathrm{OH}$ reactivity $\mathrm{SAR}^{6}{ }^{6}$ The reduced $\mathrm{OH}$ reactivity leads to longer atmospheric lifetimes. Increased fluorination also leads to stronger infrared absorption in the Earth's atmospheric window and greater well-mixed REs. The combination of low reactivity and enhanced RE leads to greater GWPs.

Notable exceptions to this general behavior are HFCs that contain a $\mathrm{CH}_{3}$ terminal group bonded to a perfluorinated moiety. Two examples are $\mathrm{CH}_{3} \mathrm{CF}_{3}$ (HFC-143a) and 
$\mathrm{CH}_{3} \mathrm{CF}_{2} \mathrm{CF}_{3}$ (HFC-245cb) for which there are experimental data and recommendations given in the WMO ozone assessment. ${ }^{1}$ Although HFC-143a is not the most fluorinated C2 HFC, it has the longest atmospheric lifetime and largest GWP of all the C2 HFCs. Similarly, HFC-245cb has the second highest atmospheric lifetime and GWP of the C3 HFCs (this holds true for both $\operatorname{GWP}(20)$ and $\operatorname{GWP}(100)$ ). This behavior is also evident for the $\mathrm{C} 4$ linear and branched molecules. There are experimentally based metrics for only one C4 linear molecule, $\mathrm{CHF}_{2} \mathrm{CF}_{2} \mathrm{CF}_{2} \mathrm{CF}_{3}$ (HFC-329p), so the comparisons discussed here are mostly based on the data from this study. The $\mathrm{C} 4$ linear molecule with the longest atmospheric lifetime and largest GWP is $\mathrm{CF}_{3} \mathrm{CHFCF}_{2} \mathrm{CF}_{3}$ (HFC-329me), whereas the molecule with the third largest lifetime and GWP is $\mathrm{CH}_{3} \mathrm{CF}_{2} \mathrm{CF}_{2} \mathrm{CF}_{3}$ (HFC-347mcc), though we will note that the lifetime and GWP are only slightly larger than for $\mathrm{CHF}_{2} \mathrm{CF}_{2} \mathrm{CF}_{2} \mathrm{CF}_{3}$ (HFC-329p), which has metrics in Table 3 taken from WMO. For the C4 branched HFCs, there are no molecules included in WMO. In this case, the HFC with the largest lifetime and GWP is $\mathrm{CF}_{3} \mathrm{CH}\left(\mathrm{CF}_{3}\right) \mathrm{CF}_{3}$ (HFC- $b$ $329 \mathrm{mz}$ ) and the molecule with the second largest lifetime and GWP is $\mathrm{CH}_{3} \mathrm{CF}\left(\mathrm{CF}_{3}\right) \mathrm{CF}_{3}$ (HFC-b-347mym). This is, again, consistent with the observation that a terminal $\mathrm{CH}_{3}$ group attached to a perfluorinated moiety leads to a greater lifetime and GWP.

A second observation is that the atmospheric lifetime is more important than the RE for a group of HFCs with a similar number of $\mathrm{C}$ and $\mathrm{F}$ atoms. That is, the $\mathrm{OH}$ reactivity has a stronger dependence on fluorination than the infrared absorption spectrum. For the $\mathrm{C} 2 \mathrm{HFCs}$, the lifetime adjusted $\mathrm{RE}$ is largest for $\mathrm{CHF}_{2} \mathrm{CF}_{3}$ (HFC-125), 0.23. However, as noted above the $\mathrm{C} 2$ molecule with the largest GWP is $\mathrm{CH}_{3} \mathrm{CF}_{3}$ (HFC-143a), which has a RE of 0.16. For the C3 HFCs, the molecule with the second largest GWP, $\mathrm{CH}_{3} \mathrm{CF}_{2} \mathrm{CF}_{3}$ (HFC$245 \mathrm{cb}$ ), has a RE of 0.24 , which is tied for only the fourth highest RE for all of the C3 HFCs. This trend continues for the C4 HFCs. The linear C4 HFC with the third highest GWP, $\mathrm{CH}_{3} \mathrm{CF}_{2} \mathrm{CF}_{2} \mathrm{CF}_{3}$ (HFC-347mcc), has a $\mathrm{RE}$ of 0.25 , and this is not even in the top ten REs for the linear C4 HFCs. For the branched $\mathrm{C} 4 \mathrm{HFCs}$, the molecule with the second largest GWP, $\mathrm{CH}_{3} \mathrm{CF}\left(\mathrm{CF}_{3}\right) \mathrm{CF}_{3}$ (HFC-b-347mym), has a $\mathrm{RE}$ of 0.29 , and this is only the ninth largest RE for the branched $\mathrm{C} 4$ HFCs. Previously, Bera et al. ${ }^{18}$ focused on the RE of HFCs as an important component that industry should consider when deciding what particular HFC to use in an industrial process. The data in Tables 1-4 support their assertion, but also indicates that the HFC atmospheric lifetime is a more important aspect to consider.

It is evident from an examination of the metrics given in WMO for the C3 HFCs in Table 2 that for HFCs with the same empirical chemical formula, i.e., isomers, those that do not have $\mathrm{H}$ atoms on the terminal $\mathrm{C}$ atoms have longer lifetimes and larger GWPs. There is one exception as noted above when there are three $\mathrm{H}$ atoms and all on a terminal $\mathrm{C}$, i.e., a $\mathrm{CH}_{3}$ group. Consider for example the empirical formula $\mathrm{C}_{2} \mathrm{H}_{2} \mathrm{~F}_{6} . \mathrm{CF}_{3} \mathrm{CH}_{2} \mathrm{CF}_{3}$ (HFC-236fa) has the largest atmospheric lifetime at 213 years and the largest GWP of all C3 HFCs. For the empirical formula $\mathrm{C}_{2} \mathrm{H}_{3} \mathrm{~F}_{5}$, aside from $\mathrm{CH}_{3} \mathrm{CF}_{2} \mathrm{CF}_{3}$ (HFC-245cb) discussed above, the HFC with the next largest lifetime and GWP is $\mathrm{CHF}_{2} \mathrm{CH}_{2} \mathrm{CF}_{3}$ (HFC245fa) consistent with this observation. Note also that $\mathrm{CHF}_{2} \mathrm{CH}_{2} \mathrm{CF}_{3}$ (HFC-245fa) is tied for the largest $\mathrm{RE}$ for the $\mathrm{C}_{2} \mathrm{H}_{3} \mathrm{~F}_{5}$ HFCs, consistent with the observations of Bera et al. ${ }^{2,18}$ This trend continues for the C4 HFCs studied here, where the linear $\mathrm{C} 4 \mathrm{HFC}$ with the longest lifetime by far and the largest GWP is $\mathrm{CF}_{3} \mathrm{CH}_{2} \mathrm{CF}_{2} \mathrm{CF}_{3}$ (HFC-338mf). However, to some extent this is to be expected, since, as noted earlier, most of the atmospheric lifetimes for the C4 HFCs are computed in this study using the $\mathrm{OH}$ reaction rate coefficients determined from the structure activity relationship (SAR) developed by DeMore. ${ }^{6}$

In summary, the larger the number of $\mathrm{F}$ atoms in a particular HFC, the greater the likelihood that HFC will possess a longer atmospheric lifetime, a large RE, and thus a large GWP. However, how the $\mathrm{F}$ atoms are distributed around the HFC, i.e., isomers, will determine how large the RE is, but more importantly, how long the atmospheric lifetime is, and ultimately the GWP.

\section{CONCLUSIONS}

In this study, policy relevant metrics have been provided for HFC compounds with fewer than 5 carbon atoms (153 compounds in total) most of which lack direct experimentally derived metric values. Tables $1-4$ summarize the results from this study and detailed datasheets for the individual HFCs are provided in the Supporting Information. We have demonstrated that HFCs with different chemical formulas and the associated isomers have significantly different atmospheric lifetimes and climate metrics. That is, reliable lifetimes and climate metrics cannot be assigned based on the empirical chemical formula alone.

We have shown that both the radiative efficiency (RE) and the atmospheric lifetime of an HFC are important parameters in determining a molecule's potential environmental impact, but the atmospheric lifetime is generally more important than the RE in the GWP determination for most HFCs. To some extent, the degree of fluorination, lifetime, and RE are correlated. This is because HFCs react more readily with $\mathrm{OH}$ when they possess more $\mathrm{H}$ atoms and the $\mathrm{RE}$ is lower because the RE is mostly determined by the number of $\mathrm{C}-\mathrm{F}$ stretching vibrations, which absorb in the Earth's atmospheric infrared window. However, we have shown that there are exceptions, since HFCs that have a $\mathrm{CH}_{3}$ terminal group bonded to essentially a perfluorinated moiety lead to an HFC with a very long atmospheric lifetime and a large GWP, even if the RE of that particular HFC is not as large as some of the others in its class (isomers). For example, for the C4 HFCs, $\mathrm{CH}_{3} \mathrm{CF}_{2} \mathrm{CF}_{2} \mathrm{CF}_{3}$ (HFC-347mcc) exhibits a larger GWP and longer lifetime than all but two linear $\mathrm{C} 4 \mathrm{HFCs}$, but its $\mathrm{RE}$ is not even in the top ten for the linear C4 HFCs.

Many of the C3 and most of the C4 HFC atmospheric lifetimes and climate metrics reported here were determined using the computational methods described herein: that is, a structure activity relationship (SAR) based approach for determining $\mathrm{OH}$ reactivity and, thus, atmospheric lifetimes and $a b$ initio MP2 computed vibrational spectra that were used in the RE determination. A comparison of the computed REs for the $\mathrm{C} 2$ and C3 HFCs with the values reported in the WMO ozone assessment ${ }^{1}$ showed that the computationally based REs exhibit $\sim 10 \%$ positive bias, in part due to the calculated infrared intensities being between $5 \%$ and $20 \%$ too large. It is important to note that the data reported herein are not a substitute for accurate detailed experimental studies of targeted compounds prior to their commercial use. However, the data reported here will help guide future experiments, particularly 
when it is difficult to separate isomers, and provide relevant information to policy decision makers.

\section{ASSOCIATED CONTENT}

\section{SI Supporting Information}

The Supporting Information is available free of charge at https://pubs.acs.org/doi/10.1021/acs.jpca.0c02679.

Summary graphs of HFC climate metrics (PDF) Data sheets for HFCs (C1) (PDF)

Data sheets for HFCs (C2) (PDF)

Data sheets for HFCs (C3) (PDF)

Data sheets for HFCs (linear-C4) (PDF)

Data sheets for HFCs (branched-C4) (PDF)

\section{AUTHOR INFORMATION}

\section{Corresponding Author}

James B. Burkholder - Earth System Research Laboratory, Chemical Sciences Division, National Oceanic and Atmospheric Administration, Boulder, Colorado 80305, United States; 다이.org/0000-0001-9532-6246; Phone: (303)-4973252; Email: James.B.Burkholder@noaa.gov

\section{Authors}

Paul Marshall - Department of Chemistry, University of North Texas, Denton, Texas 76203-5070, United States

Partha P. Bera - NASA Ames Research Center, Mountain View, California 94035, United States; BAER Institute, NASA

Research Park, Moffett Field, California 94035, United States; (1) orcid.org/0000-0003-0843-3209

Joseph S. Francisco - Department of Earth and Environmental Science and Department of Chemistry, University of

Pennsylvania, Philadelphia, Pennsylvania 19104-6316, United States; (1) orcid.org/0000-0002-5461-1486

Timothy J. Lee - NASA Ames Research Center, Mountain View, California 94035, United States; (1) orcid.org/0000-00022598-2237

Complete contact information is available at:

https://pubs.acs.org/10.1021/acs.jpca.0c02679

\section{Notes}

The authors declare no competing financial interest.

\section{ACKNOWLEDGMENTS}

This work was supported in part by NOAA's Climate Program Office Atmospheric Chemistry, Carbon Cycle, and Climate Program and NASA's Atmospheric Composition Program. Some of this work was supported by the National Aeronautics and Space Administration through the NASA Astrobiology Institute under Cooperative Agreement Notice NNH13ZDA017C issued through the Science Mission Directorate.

\section{REFERENCES}

(1) WMO (World Meteorological Organization). Scientific Assessment of Ozone Depletion: 2018, Global Ozone Research and Monitoring Project-Report No. 58; Geneva, Switzerland, 2018; 588 pp.

(2) Bera, P. P.; Francisco, J. S.; Lee, T. J. Identifying the molecular origin of global warming. J. Phys. Chem. A 2009, 113, 12694-12699.

(3) Kokkila, S. I.; Bera, P. P.; Francisco, J. S.; Lee, T. J. A group increment scheme for infrared absorption intensities of greenhouse gases. J. Mol. Struct. 2012, 1009, 89-95.

(4) WMO (World Meteorological Organization). Scientific Assessment of Ozone Depletion: 2014, Global Ozone Research and Monitoring
Project-Report No. 55 416, Geneva, Switzerland, 2014; http://www. wmo.int/pages/prog/arep/gaw/ozone_2014/ozone_asst_report. html.

(5) Burkholder, J. B.; Sander, S. P.; Abbatt, J.; Barker, J. R.; Huie, R. E.; Kolb, C. E.; Kurylo, M. J.; Orkin, V. L.; Wilmouth, D. M.; Wine, P. H. Chemical Kinetics and Photochemical Data for Use in Atmospheric Studies, Evaluation No. 18; JPL Publication 15-10; Jet Propulsion Laboratory, Pasadena, 2015; http://jpldataeval.jpl.nasa.gov.

(6) DeMore, W. B. Experimental and estimated rate constants for the reactions of hydroxyl radicals with several halocarbons. J. Phys. Chem. 1996, 100, 5813-5820.

(7) Papanastasiou, D. K.; Beltrone, A.; Marshall, P.; Burkholder, J. B. Global warming potential estimates for the $\mathrm{C}_{1}-\mathrm{C}_{3}$ hydrochlorofluorocarbons (HCFCs) included in the Kigali Amendment to the Montreal Protocol. Atmos. Chem. Phys. 2018, 18, 6317-6330.

(8) Baasandorj, M.; Fleming, E. L.; Jackman, C. H.; Burkholder, J. B. $\mathrm{O}\left({ }^{1} \mathrm{D}\right)$ kinetic study of key ozone depleting substances and greenhouse gases. J. Phys. Chem. A 2013, 117, 2434-2445.

(9) Dunning, T. H. Gaussian basis functions for use in molecular calculations. I. Contraction of $(9 \mathrm{~s} 5 \mathrm{p})$ atomic basis sets for the firstrow atoms. J. Chem. Phys. 1970, 53, 2823-2833.

(10) Dunning, T. H. Gaussian basis functions for use in molecular calculations. IV. The representation of polarization functions for the first row atoms and hydrogen. J. Chem. Phys. 1971, 55, 3958-3966.

(11) Lee, T. J.; Schaefer, H. F. Systematic study of molecular anions within the self-consistent-field approximation: $\mathrm{OH}-, \mathrm{CN}^{-}, \mathrm{C}_{2} \mathrm{H}^{-}$, $\mathrm{NH}_{2}{ }^{-}$, and $\mathrm{CH}_{3}{ }^{-}$. J. Chem. Phys. 1985, 83, 1784-1794.

(12) Huzinaga, S. Gaussian-type functions for polyatomic systems. I. J. Chem. Phys. 1965, 42, 1293-1302.

(13) Simandiras, E. D.; Amos, R. D.; Handy, N. C.; Lee, T. J.; Rice, J. E.; Remington, R. B.; Schaefer, H. F. Second-order perturbation theory and configuration interaction theory applied to medium sized molecules: Cyclopropane, ethyleneimine, ethylene oxide, fluoroethane and acetaldehyde. J. Am. Chem. Soc. 1988, 110, 1388-1393.

(14) Breidung, J.; Schneider, W.; Thiel, W.; Lee, T. J. The vibrational frequencies of difluoroethyne. J. Chem. Phys. 1992, 97, 3498-3499.

(15) Shao, Y.; Gan, Z.; Epifanovsky, E.; Gilbert, A. T. B.; Wormit, M.; Kussmann, J.; Lange, A. W.; Behn, A.; J, D.; Feng, X.; et al. Advances in molecular quantum chemistry contained in the Q-Chem 4 program package. Mol. Phys. 2015, 113, 184-215.

(16) Hodnebrog, Ø.; Etminan, M.; Fuglestvedt, J. S.; Marston, G.; Myhre, G.; Nielsen, C. J.; Shine, K. P.; Wallington, T. J. Global warming potentials and radiative efficiencies of halocarbons and related compounds: A comprehensive review. Rev. Geophys. 2013, 51, $300-378$

(17) IPCC Climate Change 2013: The Physical Science Basis. Contribution of Working Group 1 to the Fifth Assessment Report of the Intergovernmental Panel on Climate Change, Stocker, T. F.; Qin, D.; Plattner, G.-K.; Tignor, M.; Allen, S.K.; Boschung, J.; Nauels, A.; Zia, Y.; Bex, V.; Midgley, P.M., Eds.; Cambridge University Press: Cambridge, United Kingdom and New York, NY, USA, 2013.

(18) Bera, P. P.; Francisco, J. S.; Lee, T. J. Design strategies to minimize the radiative efficiency of global warming molecules. Proc. Natl. Acad. Sci. U. S. A. 2010, 107, 9049-9054. 